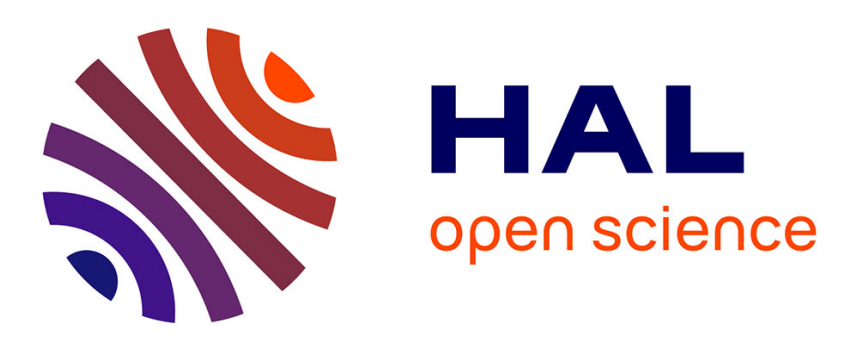

\title{
Online mobile C-arm calibration using inertial sensors: a preliminary study in order to achieve CBCT
}

\author{
Imane Lemammer, Olivier J.J. Michel, H Ayasso, Steeve Zozor, Guillaume
}

Bernard

\section{- To cite this version: \\ Imane Lemammer, Olivier J.J. Michel, H Ayasso, Steeve Zozor, Guillaume Bernard. Online mobile C-arm calibration using inertial sensors: a preliminary study in order to achieve CBCT. International Journal of Computer Assisted Radiology and Surgery, 2020, 15, pp.213-224. 10.1007/s11548-019- 02061-6 . hal-02357073}

\section{HAL Id: hal-02357073 https://hal.science/hal-02357073}

Submitted on 16 Nov 2020

HAL is a multi-disciplinary open access archive for the deposit and dissemination of scientific research documents, whether they are published or not. The documents may come from teaching and research institutions in France or abroad, or from public or private research centers.
L'archive ouverte pluridisciplinaire HAL, est destinée au dépôt et à la diffusion de documents scientifiques de niveau recherche, publiés ou non, émanant des établissements d'enseignement et de recherche français ou étrangers, des laboratoires publics ou privés. 


\title{
International Journal of Computer Assisted Radiology and Surgery Online calibration of a mobile $\mathrm{C}$-arm using inertial sensors A feasibility study in order to achieve CBCT --Manuscript Draft--
}

Manuscript Number:

Full Title:

Article Type:

Keywords:

Corresponding Author:
CARS-D-19-00062

Online calibration of a mobile $\mathrm{C}$-arm using inertial sensors

A feasibility study in order to achieve CBCT

Original Article

Mobile C-arm; СBCT; pose estimation; Online calibration; Gyroscope;

Accelerometer; Error propagation

Imane Lemammer

GIPSA-Lab

Saint-Martin-d'Hères, FRANCE
Corresponding Author Secondary Information:

Corresponding Author's Institution:

\section{Corresponding Author's Secondary} Institution:

\section{First Author:}

First Author Secondary Information:

Order of Authors:

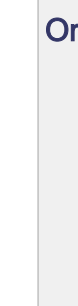

Order of Authors Secondary Information:

\section{Funding Information:}

Imane Lemammer

Imane Lemammer

Olivier Michel

Hacheme Ayasso

Steeve Zozor

Guillaume Bernard

\begin{abstract}
:
Purpose: Cone beam computed tomography (CBCT) became increasingly popular over the last years. In fact, it allows more accurate diagnosis and treatment planning with, potentially, a lower effective radiation dose. However, volume reconstruction algorithms require a very precise knowledge of the imaging geometry. Due to mechanical instabilities which significantly alter the source and the detector from a regular circular trajectory and lead to non-reproducible motions from run to run, mobile $\mathrm{C}$-arms are incompatible with existing tomography algorithms. Therefore, $\mathrm{C}$-arm on-line calibration is essential in order to achieve an accurate volume reconstruction.

Methods: We present an on-line calibration method for mobile C-arms. It is based on tracking the detector and the X-ray source of the C-arm using three-axis gyroscopes and accelerometers. It aims to be precise, non-invasive and suitable for every C-arm. The performance of the calibration algorithm is evaluated in regard to the precision of the measurements and to whether or not dynamic models of the C-arm are considered. In addition, we present an algorithm which allows us to propagate the errors from the pose (position and orientation) estimates to 2D projections on the detector plane. Thus, we can evaluate the impact of those errors on the acquired image.

Results: The experiments are conducted on an experimental platform. The reached accuracy is $\approx 0.3^{\circ}$ for orientation and $\approx 3.2 \mathrm{~mm}$ for position. These errors propagates as an error of a few millimeters for the $2 \mathrm{D}$ projections on the detector plane.
\end{abstract}

Association Nationale de la Recherche et Mrs. Imane Lemammer de la Technologie

Thales Group Mrs. Imane Lemammer 
Conclusions: the required angle accuracy by our CBCT algorithms is reached. However, several improvement to dynamic model and to estimation method are needed to achieve the position precision required by CBCT. Furthermore, we notice that the detector angles and the source-to-detector and source-to-reconstruction distances impact the projections less significantly than the in-plane translations of the X-ray source and the detector. 
Noname manuscript No.

(will be inserted by the editor)

\title{
Online calibration of a mobile C-arm using inertial sensors
}

\section{A feasibility study in order to achieve CBCT}

\author{
Imane Lemammer • Olivier Michel • \\ Hacheme Ayasso · Steeve Zozor . \\ Guillaume Bernard
}

Received: date / Accepted: date

\begin{abstract}
Purpose Cone beam computed tomography (CBCT) became increasingly popular over the last years. In fact, it allows more accurate diagnosis and treatment planning with, potentially, a lower effective radiation dose. However, volume reconstruction algorithms require a very precise knowledge of the imaging geometry. Due to mechanical instabilities which significantly alter the source and the detector from a regular circular trajectory and lead to non-reproducible motions from run to run, mobile $\mathrm{C}$-arms are incompatible with existing tomography algorithms. Therefore, $\mathrm{C}$-arm on-line calibration is essential in order to achieve an accurate volume reconstruction.

Methods We present an on-line calibration method for mobile C-arms. It is based on tracking the detector and the X-ray source of the $\mathrm{C}$-arm using three-axis gyroscopes and accelerometers. It aims to be precise, non-invasive and suitable for every C-arm. The performance of the calibration algorithm is evaluated in regard to the precision of the measurements and to whether or not dynamic models of the $\mathrm{C}$-arm are considered. In addition, we present an algorithm which allows us to propagate the errors from the pose (position and orientation) estimates to $2 \mathrm{D}$ projections on the detector plane. Thus, we can evaluate the impact of those errors on the acquired image.

Results The experiments are conducted on an experimental platform. The reached accuracy is $\approx 0.3^{\circ}$ for orientation and $\approx 3.2 \mathrm{~mm}$ for position. These errors propagates as an error of a few millimeters for the $2 \mathrm{D}$ projections on the detector plane.
\end{abstract}

Conclusions the required angle accuracy by our CBCT algorithms is reached. However, several improvement to dynamic model and to estimation method are needed to achieve the position precision required by CBCT. Furthermore,

I. Lemammer · O. Michel · H. Ayasso · S. Zozor

Univ. Grenoble Alpes, CNRS, Grenoble INP, GIPSA-Lab, F-38000 Grenoble, France

Tel.: + 33(0)476827131

I. Lemammer · G. Bernard

Thales AVS France, 460 Rue du Pommarin, 38430 Moirans, France 
we notice that the detector angles and the source-to-detector and source-toreconstruction distances impact the projections less significantly than the inplane translations of the X-ray source and the detector.

Keywords Mobile C-arm · CBCT · Pose estimation · Online calibration · IMU · Error propagation

\section{Introduction}

Cone beam computed tomography (CBCT) offers important advances in numerous medical procedures including orthopedic and trauma surgery [12]. For tomographic reconstruction, a sequence of images is captured during the Carm rotation around the patient and the precise projection geometry has to be determined for each image (submillimetric precision). Many fixed angiography systems are designed to allow 3D tomography [15]. However, those systems are expensive and their use is mainly restricted to major surgery.

Meanwhile, thanks to their size, flexibility and price, mobile C-arms are a widely used tool in trauma and orthopedic surgery. They are fluoroscopic imaging devices composed of a C-shaped arm which connects an X-ray source and a detector. However, they suffer from mechanical instabilities and gravitational effects during orbital rotation which significantly alter the source and the detector from a regular circular trajectory [21] and lead to non-reproducible motions from run to run. As there is a significant deterioration of the reconstructed image quality when the real poses of the scan differ from the poses of the initial calibration scan [16], mobile C-arms appear to be incompatible with existing volume reconstruction algorithms.

The geometric characterization of a C-arm consists in the determination of a projection matrix described by three intrinsic parameters and six extrinsic parameters [14]. The classical approach to acquire the projection geometry of the C-arm is off-line calibration [18,4]. It assumes the reproducibility of the $\mathrm{C}$-arm movement. Due to the geometric non-idealities of the source-detector orbit, maximum departures from the average semicircular orbit of the projection matrix parameters is important, even short-term $(\approx 4 h)$ : up to $16 \mathrm{~mm}$ for distances and to $2^{\circ}$ for angles for a modern mobile C-arm [5]. Therefore, an on-line recovery of the projection geometry seems necessary to conduct $\mathrm{CBCT}$ with mobile C-arms.

The first approach towards on-line calibration consists in using fiducial markers $[17,10]$. It is inexpensive, potentially precise $(1-3 \mathrm{~mm}$ translation accuracy and $1-2^{\circ}$ orientation accuracy) $[19,23]$ and applicable for every C-arm. However, the markers based on-line calibration is invasive and the markers occupy valuable place in the image. Another method of on-line calibration involves external tracking of the mobile C-arm [13]. Optical cameras have been used to track the $\mathrm{C}$-arm pose accurately $(3.1 \mathrm{~mm} \pm 1.3 \%)$. However, the drawback is the need of a precise setup and direct line sight between the mobile C-arm and the camera, leading to major modification in the process of performing surgical acts. 
Even if accelerometers have already been used as tilt sensors for non-motorized C-arms $[8,22]$, a precise estimation of the attitude of the X-ray source and the detector of a mobile $\mathrm{C}$-arm in a surgical environment using inertial sensors, eventually combined with additional models, is still to be made.

In this paper, we are going to discuss the implementation feasibility of a precise and affordable on-line calibration approach for existing mobile C-arms using inertial sensors (gyroscopes and accelerometers). It has to be non-invasive and suitable for every mobile C-arm even those with highly non-reproducible trajectories. Moreover, it should not significantly modify the surgical routine. We are also going to introduce an algorithm which propagates the errors from the pose estimates of the X-ray source and the detector to the $2 \mathrm{D}$ projections and evaluate the performance of our calibration algorithm.

\section{Material and methods}

\subsection{Mobile C-arms}

Geometry description: The C-arm geometry is detailed in Fig. 1 and the used notations are described in Tab. 1. This geometry is defined by four righthanded Cartesian coordinate systems: the navigation frame $\{w\}$ which is a fixed frame around which the C-arm gantry rotates and within which the reconstruction volume is computed, the real detector frame $\{d\}$, the virtual detector frame $\left\{d^{\prime}\right\}$ which has the same orientation as $\{d\}$ but is centered at ${ }^{w} o$, and the source frame $\{s\}$.
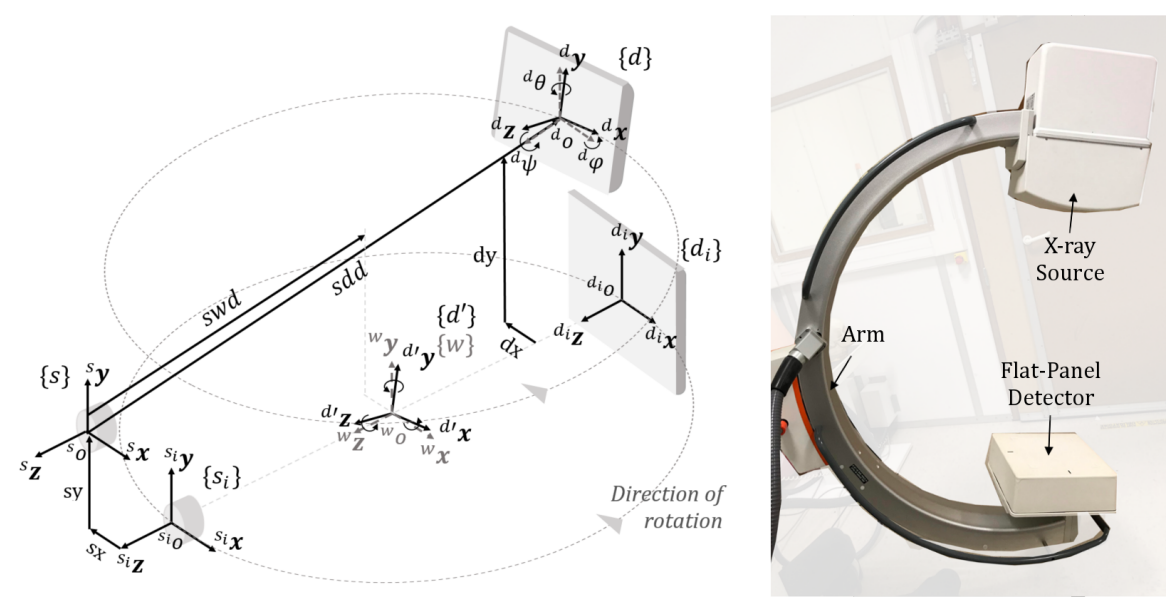

Fig. 1 (Right) The mobile C-arm. (Left) Mobile C-arm geometry and motion compared to the motion of a perfect circular geometry represented by $\left\{d_{i}\right\}$ and $\left\{s_{i}\right\}$. The mobile $\mathrm{C}$-arm is provided with four coordinate systems: the reconstruction fixed frame $\{w\}$, the detector frame $\{d\}$, the virtual detector frame $\left\{d^{\prime}\right\}$ and the source frame $\{s\}$. swd is the source-to-reconstruction distance and $s d d$ the source-to-detector distance. 
Table 1 Table of notations.

\begin{tabular}{ll}
\hline Notation & Meaning \\
\hline $\mathbf{a}$ & scalar \\
$\mathbf{a}$ & vector \\
$\mathbf{A}$ & matrix \\
$q$ & quaternion \\
$\dot{a}$ & time derivative of $a$ \\
$\mathbf{A}^{t} / \mathbf{a}^{t}$ & transpose of the matrix $\mathbf{A} /$ vector a \\
$\operatorname{diag}(\mathbf{a})$ & square diagonal matrix with the elements of vector a on the main diagonal \\
$\mathbf{I}$ & identity matrix of the appropriate size \\
$\{a\}$ & frame or coordinate system \\
$n$ & vector a expressed in frame $\{n\}$ \\
$\mathbf{a} k$ & vector a for the sample $k$ \\
$b$ \\
$n$ & unit quaternion describing the rotation from frame $\{n\}$ to frame $\{b\}$ \\
${ }_{n} \mathbf{R}$ & rotation matrix of frame $\{b\}$ with respect to frame $\{n\}$ \\
\hline
\end{tabular}

C-arm motion: In order to compute $\mathrm{CBCT}$, multiple $2 \mathrm{D}$ projections are acquired under a continuous rotation of the C-arm gantry. In our study, the motion of the gantry about a single axis ${ }^{w} \mathbf{y}$ is studied. This rotation is usually operated manually. Due to the imperfections of machine manufacturing, to the gravitational effects and to the mechanical flex, the trajectory of the detector and the X-ray source deviate significantly from a circular path. The gantry may be deviated in 3 translation directions and 3 rotation directions due to the crude tilting of the detector. These perturbations are non-reproducible.

Given its maximum tilt angles, the X-ray source is supposed to be isotopic. Thus, its orientation can be neglected for the calculation of the projection matrix Seq. 2.4. However, the orientation of the X-ray source must be determined in order to estimate its position from the accelerometer measurements. Also, the C-arms support armature is assumed to be fixed with respect to the reconstruction frame $\{w\}$.

\subsection{Inertial sensors}

An inertial measurement unit (IMU) combining a three-axis gyroscope and a three-axis accelerometer is mounted on both the detector and the source of the $\mathrm{C}$-arm. The X-ray source and the detector poses are supposed to be independent. Therefore, two separate and similar studies will be conducted for the source and for the detector. We are going to develop the problem of estimating the relative pose of the moving detector frame $\{d\}$ at time $k \Delta t$ ( $\Delta t$ is the sampling period of the sensor and $k$ the sample id) with respect to the reconstruction frame $\{w\}$. The problem is illustrated in Fig. 2. The same reasoning applies to the X-ray source.

Reconstruction frame $\{w\}$ : frame where the 3D tomographic reconstruction is conducted. As the measurements are acquired over short acquisition times $(<1 \mathrm{~min})$, the reconstruction frame is supposed to be fixed with respect to the earth. 

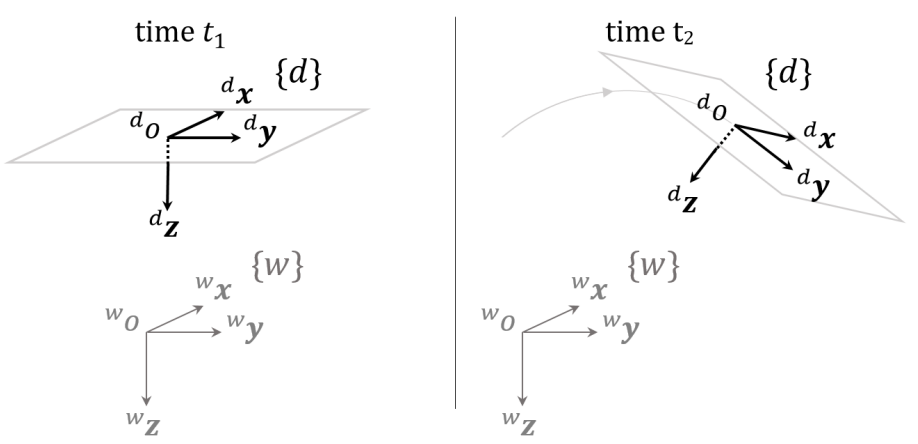

Fig. 2 The inertial sensors measure acceleration and angular velocity with respect to the reconstruction frame $\{w\}$. The sensors frames are confounded with the detector frame $\{d\}$.

Detector frame $\{d\}$ : frame of the moving IMU. Its origin is supposed to be located at the center of the accelerometer triad and aligned to the casing. All the inertial measurements are resolved in this frame.

\subsubsection{Measurement models}

Gyroscope measurement model: The gyroscope gives us the angular velocities of the detector frame $\{d\}$ with respect to the reconstruction frame $\{w\}$ expressed in the detector frame $\{d\}$. The angular velocities measured by the three-axis gyroscope at time $k \Delta t$ are denoted by the three-dimensional vector ${ }_{n}^{b} \boldsymbol{\omega}_{k}$. The three-axis gyroscope measurements are affected by bias and noise. We assume that the chosen gyroscopes ensure an adequate bandwidth for the motion. The gyroscopes are usually sensitive to linear acceleration (or $g$-sensitivity), vibration rectification (or $g^{2}$-sensitivity) [7] and temperature fluctuations. However, the C-arm operating time is usually short $(<1 \mathrm{~min})$ and the typical deviation of temperature is about $0.01^{\circ} \mathrm{C}$ within similar duration which makes temperature fluctuations negligible. Acceleration sensitivity is also negligible in our case, the gyroscope measurements $\mathbf{y}_{\omega, k}$ are expressed as

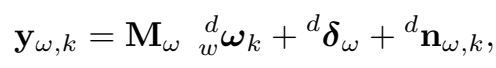

where:

$-\mathbf{M}_{\omega}$ is the gyroscope measurements scaling factors and misalignment matrix,

$-{ }^{d} \boldsymbol{\delta}_{\omega}$ is the gyroscope constant bias,

$-{ }^{d} \mathbf{n}_{\omega, k}$ is the noise in the gyroscope. It is modeled as an additive zero-mean Gaussian noise with a covariance matrix: $\boldsymbol{\Sigma}_{\omega}=\operatorname{diag}\left(\sigma_{\omega, x}^{2}, \sigma_{\omega, y}^{2}, \sigma_{\omega, z}^{2}\right)$. We write

$$
{ }^{d} \mathbf{n}_{\omega, k} \sim \mathcal{N}\left(0, \boldsymbol{\Sigma}_{\omega}\right) .
$$


Accelerometer measurement model: An ideal accelerometer measures the physical acceleration of an object. The physical acceleration is the sum of the relative acceleration ${ }^{w} \mathbf{a}$ and the contribution of the physical forces applied to the accelerometer:

$$
{ }^{w} \mathbf{a}_{m}={ }^{w} \mathbf{a}+{ }^{w} \mathbf{a}_{g}+{ }^{w} \mathbf{a}_{c}
$$

where:

$-{ }^{w} \mathbf{a}_{m}$ is the acceleration measured by the three-axis accelerometer,

$-{ }^{w} \mathbf{a}$ is the relative acceleration which results of the movement of the body, $-{ }^{w} \mathbf{a}_{g}$ is the local acceleration due to gravity,

$-{ }^{w} \mathbf{a}_{c}$ is the Coriolis acceleration.

A numerical application shows that ${ }^{w} \mathbf{a}_{c} \approx 10^{-4} \mathrm{~m} / \mathrm{s}^{2}$. Because this value is lower than the accelerometer noise level, we consider that ${ }^{w} \mathbf{a}_{c}=0$. Following the same reasoning as for the gyroscope, we express the accelerometer measurements $\mathbf{y}_{a, k}$ as

$$
\mathbf{y}_{a, k}=\mathbf{M}_{a} \underset{w}{d} \mathbf{R}_{k}\left({ }^{w} \mathbf{a}_{k}-\mathbf{g}\right)+{ }^{d} \boldsymbol{\delta}_{a}+{ }^{d} \mathbf{n}_{a, k},
$$

where:

$-\mathbf{M}_{a}$ is the accelerometer measurements scaling factors and misalignment matrix,

$-{ }_{w}^{d} \mathbf{R}_{k}$ is the rotation matrix from $\{w\}$ to $\{d\}$,

- $\mathbf{g}$ is the gravitational force, here directed along the ${ }^{w} \mathbf{y}$-axis,

$-{ }^{d} \boldsymbol{\delta}_{a}$ is the accelerometer bias,

$-{ }^{d} \mathbf{n}_{a, k}$ is the noise in the accelerometer. It is modeled as an additive zeromean Gaussian noise with a covariance matrix: $\boldsymbol{\Sigma}_{a}=\operatorname{diag}\left(\sigma_{a, x}^{2}, \sigma_{a, y}^{2}, \sigma_{a, z}^{2}\right)$

\subsection{Attitude estimation}

\subsubsection{Dead-Reckoning}

One of the simplest methods for pose estimation is dead reckoning (DR). It is the process of estimating the present position and orientation of an object, based on a previously known position and orientation. This method is based on inertial sensors measurements only. DR can make very accurate estimates if the speeds and directions of the object are accurately measured at all instants. However, inertial sensors measurements are noisy and biased Seq. 2.2.1. Hence, the DR estimates are subject to significant errors.

Principle: Since the measurements are resolved in the detector frame $\{d\}$, the position ${ }^{d} \mathbf{p}$ can be evaluated from the measured acceleration ${ }^{d} \mathbf{a}_{m}$ using double integration. As well as, the quaternion for orientation ${ }_{w}^{d} q$ can be evaluated by integrating the angular velocity $\underset{w}{d} \boldsymbol{\omega}$. This algorithm is illustrated in Fig. 3. The accuracy of DR estimates can be increased significantly by taking into account suitable dynamic models. Therefore, a method which compares the inertial sensors readings with a pose predicted using the current estimate and simple dynamic models is also implemented. 


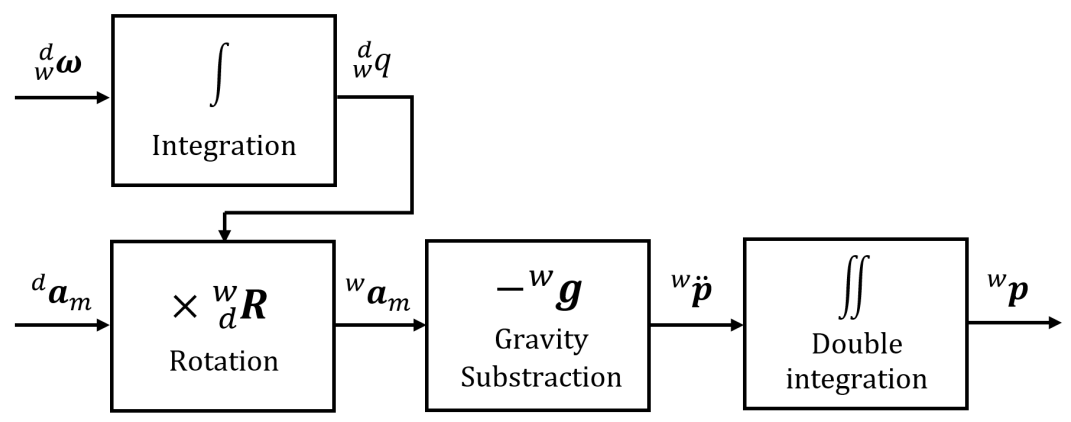

Fig. 3 The block diagram of DR implementation for IMUs.

\subsubsection{Extended Kalman filtering}

In order to simplify the formulation of the pose estimation problem, we drop the frame subscripts and superscripts, for example ${ }^{w} \mathbf{a}_{k}$ is written $\mathbf{a}_{k}$ and ${ }_{w}^{d} \boldsymbol{\omega}_{k}$ is written $\boldsymbol{\omega}_{k}$.

Principle: The Kalman filter [1] (KF) operates in two distinct phases: prediction and correction. In the prediction phase, the detector's current pose is predicted from the old pose according to the dynamic models. A new pose estimate and a new covariance are calculated. Next, in the correction phase, the inertial sensors measurements and their uncertainties are used to correct the prediction. These uncertainties and the predicted covariance (from the previous phase) determines how much the measurements will affect the prediction. The Extended Kalman Filter (EKF) is an extension of the traditional Kalman filter to non linear systems such as rotations. The principle of the Kalman filtering is described in the diagram Fig. 4. As second order Taylor expan-

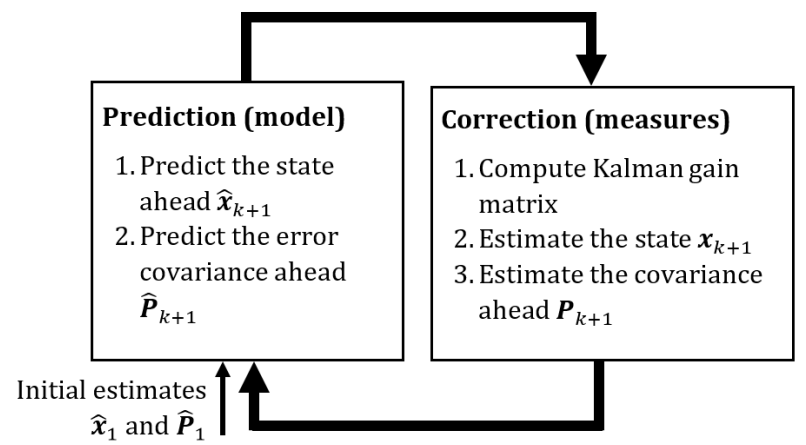

Fig. 4 the $\mathrm{KF}$ is an algorithm that uses measurements and process models to produce estimates that tend to be more accurate than those based on the measurements only. This is a two-step algorithm: prediction and correction. It also predicts the estimate uncertainties. 
sion lead to a more accurate estimation in our case than first order, we use second order Taylor expansion to linearize the EKF models. Over our short acquisition time $(<1 \mathrm{~min})$, it also appears to be more efficient to estimate orientation based on the angular velocity measured by the gyroscope only. The inverse estimated orientation is then used to rotate the detector frame $\{d\}$ in order to estimate the position into the reconstruction frame $\{w\}$. Since the Coriolis acceleration is negligible and the angular rate is independent from linear acceleration Seq. 2.2.1, we implement two separate EKF for orientation and position estimation.

Quaternions: The implemented EKF fuses attitude estimations in quaternion form. Quaternions [11] are widely used in orientation estimation algorithms. They have no singularities and are simpler to compose than Euler angles. Moreover, they are more compact and numerically stable than rotation matrices. A quaternion is denoted

$$
q=\left[\begin{array}{llll}
q_{0} & q_{1} & q_{2} & q_{3}
\end{array}\right]^{t} .
$$

Here, $q_{0}$ is the scalar component and $\mathbf{q}_{v}=\left[\begin{array}{lll}q_{1} & q_{2} & q_{3}\end{array}\right]^{t}$ is the vector component of the quaternion. The unit quaternion representing the rotation from frame $\{w\}$ to frame $\{d\}$ is written ${ }_{w}^{d} q$. This quaternion corresponds to the rotation matrix ${ }_{w}^{d} \mathbf{R}$. Assuming that $q={ }_{w}^{d} q$, the rotation from frame $\{d\}$ to frame $\{w\}$ can be represented by the conjugate quaternion defined as

$$
q^{*}=\left[\begin{array}{llll}
q_{0} & -q_{1} & -q_{2} & -q_{3}
\end{array}\right]^{t} .
$$

In this paper, the identity quaternion has the following components

$$
q_{I}=\left[\begin{array}{llll}
1 & 0 & 0 & 0
\end{array}\right]^{t} .
$$

Successive rotations about local axes are composed using quaternion multiplication. For example ${ }_{i}^{d} q={ }_{w}^{d} q{ }_{i}^{w} q$ represents a rotation from frame $\{i\}$ to $\{d\}$ followed by a rotation from frame $\{w\}$ to frame $\{d\}$. Quaternion multiplication is calculated as

$$
q p=\left[\begin{array}{l}
p_{0} q_{0}-p_{1} q_{1}-p_{2} q_{2}-p_{3} q_{3} \\
p_{0} q_{1}+p_{1} q_{0}+p_{2} q_{3}-p_{3} q_{2} \\
p_{0} q_{2}-p_{1} q_{3}+p_{2} q_{0}+p_{3} q_{1} \\
p_{0} q_{3}+p_{1} q_{2}-p_{2} q_{1}+p_{3} q_{0}
\end{array}\right] .
$$

Orientation estimation dynamic model: The state vectors for orientation estimation $\mathbf{x}_{k}$ is

$$
\mathbf{x}_{k}=\left[\begin{array}{ll}
q_{k} & \boldsymbol{\omega}_{k}
\end{array}\right]^{t} .
$$

The continuous process model equation is described by

$$
\dot{q}=\frac{1}{2} \Omega q,
$$


with $\boldsymbol{\Omega}$ the relative rotation matrix written

$$
\boldsymbol{\Omega}=\left[\begin{array}{cc}
0 & \boldsymbol{\omega}^{t} \\
-\boldsymbol{\omega} & -\boldsymbol{\omega}^{\times}
\end{array}\right]
$$

where $\boldsymbol{\omega}=\left[\omega_{x} \omega_{y} \omega_{z}\right]^{t}$ is the angular rate for the ${ }^{d} \mathbf{x},{ }^{d} \mathbf{y}$ and ${ }^{d} \mathbf{z}$-axis of the detector frame $\{d\}$ and $\boldsymbol{\omega}^{\times}$is the angular rate skew matrix

$$
\left[\begin{array}{ccc}
0 & \omega_{z} & -\omega_{y} \\
-\omega_{z} & 0 & \omega_{x} \\
\omega_{y} & -\omega_{x} & 0
\end{array}\right]
$$

The discrete-time system process equation at time $k \Delta t$ is

$$
q_{k+1}=\exp \left(\boldsymbol{\Omega}_{k} \Delta t\right) q_{k}+\mathbf{u}_{k}
$$

Here, $\Delta t$ is the system sampling period and $\mathbf{u}_{k}$ is the orientation estimation process noise. As we work with high sample rates, $\Omega_{k} \Delta t$ is supposed small between two consecutive samples. Hence, we can approximate $\exp \left(\boldsymbol{\Omega}_{k} \Delta t\right)$ using the second order Taylor expansion,

$$
q_{k+1}=\left(\mathbf{I}+\frac{1}{2} \boldsymbol{\Omega}_{k} \Delta t-\frac{1}{2 !}\left(\frac{1}{2} \boldsymbol{\Omega}_{k} \Delta t^{2}\right)\right) q_{k}+\mathbf{u}_{k}
$$

Position estimation dynamic model: The state vectors for position estimation $\mathbf{x}_{k}^{\prime}$ is

$$
\mathbf{x}_{k}^{\prime}=\left[\begin{array}{lll}
\mathbf{p}_{k} & \mathbf{v}_{k} & \mathbf{a}_{k}
\end{array}\right]^{t},
$$

where $\mathbf{p}_{k}, \mathbf{v}_{k}$ and $\mathbf{a}_{k}$ are the positions, velocities and acceleration at time $k \Delta t$ respectively. The continuous process model equations are described by the basic continuous time dynamic spatial relations

$$
\begin{aligned}
& \mathbf{v}=\dot{\mathbf{p}}, \\
& \mathbf{a}=\dot{\mathbf{v}} .
\end{aligned}
$$

The process equations are discretized using the explicit midpoint discretization method [2] which can be summarized by the equations

$$
\begin{aligned}
& \mathbf{v}_{k+1}=\Delta f\left(t_{k}+\frac{\Delta}{2}, \mathbf{v}_{k}+\frac{\Delta}{2} \mathbf{a}_{k}\right), \\
& \mathbf{p}_{k+1}=\Delta f\left(t_{k}+\frac{\Delta}{2}, \mathbf{p}_{k}+\frac{\Delta}{2} \mathbf{v}_{k}\right) .
\end{aligned}
$$

Here, $\dot{\mathbf{p}}(t)=f(t, \mathbf{p}(t))$ and $t_{k}=t_{0}+k \Delta t$ with $t_{0}$ the initial time. The same applies for $\mathbf{v}$. To keep the dynamic model simple, we model the motion as a uniform circular motion around ${ }^{w} \mathbf{y}$. We write $\mathbf{a}=-\boldsymbol{\omega}^{2} \mathbf{p}$. 


\subsection{Projective geometry}

C-arms are modeled after a pinhole camera model [6]. The 3D projective space is represented by 4 -dimensional vectors of homogeneous coordinates $\mathbf{x}=\left[\begin{array}{llll}x_{1} & x_{2} & x_{3} & x_{4}\end{array}\right]^{t}$ where at least one of the component is nonzero.

Within this model, the X-ray projection geometry is represented by a $(3 \times 4)$ homogeneous projection matrix $\mathbf{P}$. As displayed in Fig. 5, in order to calculate the coordinates of the $2 \mathrm{D}$ projection ${ }_{d}^{d} \mathbf{p}_{2 D}$ of a $3 \mathrm{D}$ voxel ${ }_{w}^{w} \mathbf{p}_{3 D}$ on the detector plan, four changes of frames and affine transformations are operated. As

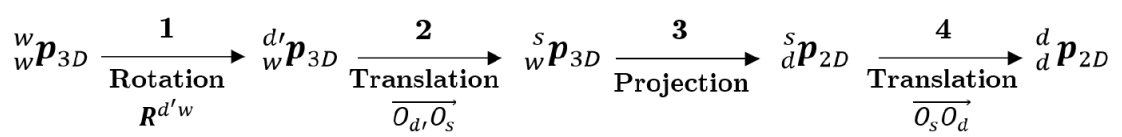

Fig. 5 To map a 2D detector image projection ${ }_{d}^{d} \mathbf{p}_{2 D}$ to the corresponding $3 \mathrm{D}$ voxel ${ }_{w}^{w} \mathbf{p}_{3 D}$ : (1) The voxel coordinates are rotated to the virtual detector coordinate system. (2) Then, they are translated to the source frame. (3) The voxel is projected. (4) Finally, the projection coordinates are expressed in the detector frame.

shown in the matrix expression (18) and Fig. 1, the C-arm projective geometry is characterized by nine parameters. Six extrinsic parameters combined in $\mathbf{E}$, which describe the position of the mobile $\mathrm{C}$-arm with respect to the reconstruction frame. These parameters are the detector orientation $\left[\begin{array}{lll}d_{\phi} & { }^{d} \theta & { }^{d} \psi\end{array}\right]^{t}$ and the source position in the reconstruction frame $\left[\begin{array}{lll}s x & s y & s w d\end{array}\right]^{t}$. And also, three intrinsic parameters combined in $\mathbf{I}$, that refer to the relative distance between the source and the detector $s d d$ (focal distance) and the piercing point coordinates $\left[\begin{array}{ll}s x-d x & s y-d y\end{array}\right]^{t}$. The piercing point, Fig. 1, is the projection of the origin of the source coordinate system on the detector plane.

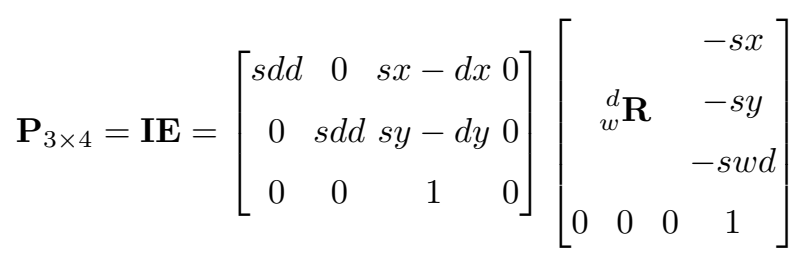

Error propagation to the 2D projections: As the inertial sensors measurements are noisy and biased Seq. 2.2.1, we calculate the projection matrix parameters with uncertainties. We denote $\delta x$ the uncertainty for $x$. The upper limit value of the uncertainty for the projection matrix $\mathbf{P}_{3 \times 4}$ is

$$
\begin{aligned}
\delta \mathbf{P}_{3 \times 4} \leq & \left|\frac{\partial \mathbf{P}}{\partial s x}\right| \delta s x+\left|\frac{\partial \mathbf{P}}{\partial s y}\right| \delta s y+\left|\frac{\partial \mathbf{P}}{\partial d x}\right| \delta d x+\left|\frac{\partial \mathbf{P}}{\partial d y}\right| \delta d y+\left|\frac{\partial \mathbf{P}}{\partial s d d}\right| \delta s d d \\
& +\left|\frac{\partial \mathbf{P}}{\partial s d d}\right| \delta s w d+\left|\frac{\partial \mathbf{P}}{\partial^{d} \phi}\right| \delta^{d} \phi+\left|\frac{\partial \mathbf{P}}{\partial^{d} \theta}\right| \delta^{d} \theta+\left|\frac{\partial \mathbf{P}}{\partial^{d} \psi}\right| \delta^{d} \psi
\end{aligned}
$$


In order to calculate the partial derivatives, we assume that the geometric parameters are independent.

\section{Results}

\subsection{Experimental setting}

Our first tracking experiments are carried out a on full-size mock-up of a mobile C-arm. The mock-up displayed in Fig. 6 is a turntable rotated by a brushless motor and equipped with a wooden arm on which are located several IMUs with different characteristics.

\subsubsection{Rotated-arm}

The rotated-arm is a simple mock-up to simulate either the detector or the Xray source of a mobile $\mathrm{C}$-arm. It is an isocentric rotation system with a gantry axis slightly inclined with respect to the gravity vector. It is also subject to significant reproducible mechanical vibrations. The rotated-arm exhibits a good repeatability (up to $99 \%$ in all directions). This allows us to simulate realistic trajectories. In order to evaluate the tracking algorithms and to propagate the errors to the $2 \mathrm{D}$ projections, the experimental estimates will be compared to the corresponding simulated trajectory.

Side view

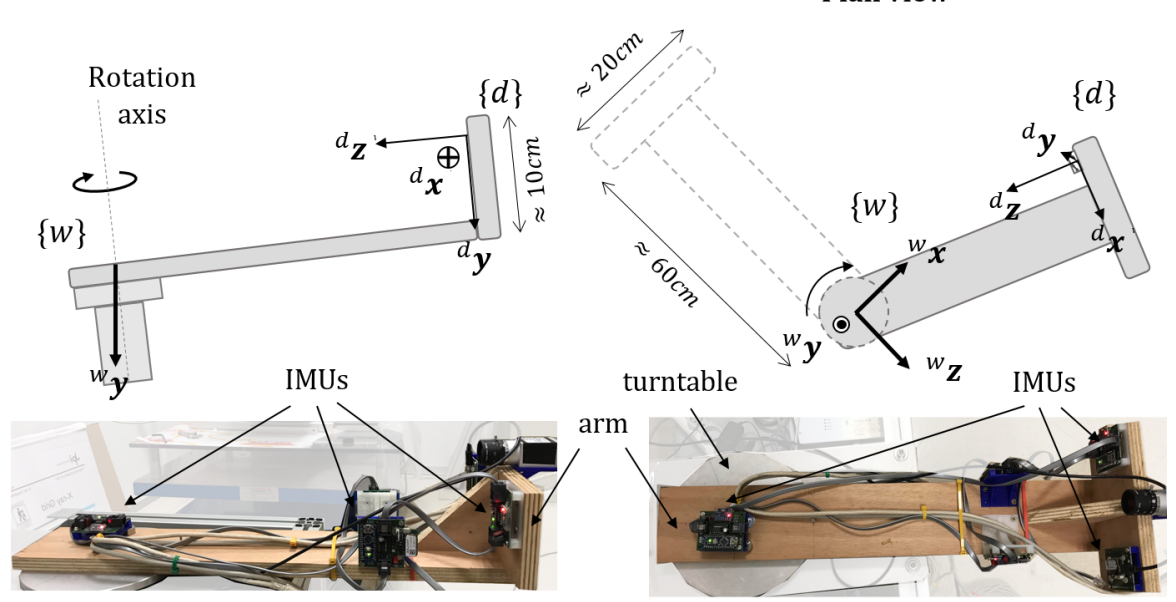

Fig. 6 The experimental rotated-arm. The mock-up aims to reproduce the behavior of the detector or the X-ray source of a mobile C-arm. The figure displays diagrams and photographs of the rotated arm. 


\subsubsection{Measurement units}

The experimental platform is equipped with two different IMUs:

The MPU6050 from TDK-Invensense [9] which is a low-cost IMU. It must be calibrated manually and is subject to significant nonlinearities. However, the MPU6050 is a flexible component with a large choice of acquisition frequencies and measuring ranges. For this IMU, the standard deviations (SD) of noise as defined in (2) are $\left[\begin{array}{lll}\sigma_{\omega, x} & \sigma_{\omega, y} & \sigma_{\omega, z}\end{array}\right]=\left[\begin{array}{lll}1.1 & 1.0 & 0.90\end{array}\right] \times 10^{-3}$ $(\mathrm{rad} / \mathrm{s})^{2}$ and $\left[\begin{array}{lll}\sigma_{a, x} & \sigma_{a, y} & \sigma_{a, z}\end{array}\right]=\left[\begin{array}{lll}36 & 30 & 40\end{array}\right] \times 10^{-3}\left(\mathrm{~m} / \mathrm{s}^{2}\right)^{2}$.

The NavChip ISNC02 from THALES-Intersense [20] which is a superior performance factory calibrated sensor with temperature and axis misalignment compensation. It returns integrated values of angular velocity and acceleration which has been previously filtered. However, it is not tunable and is provided with one acquisition frequency and fixed measurement ranges. The measurements SDs are $\left[\begin{array}{lll}\sigma_{\omega, x} & \sigma_{\omega, y} & \sigma_{\omega, z}\end{array}\right]=\left[\begin{array}{lll}0.81 & 0.94 & 0.92\end{array}\right] \times 10^{-3}$ $(\mathrm{rad} / \mathrm{s})^{2}$ and $\left[\begin{array}{lll}\sigma_{a, x} & \sigma_{a, y} & \sigma_{a, z}\end{array}\right]=\left[\begin{array}{lll}9.1 & 6.2 & 11\end{array}\right] \times 10^{-3}\left(\mathrm{~m} / \mathrm{s}^{2}\right)^{2}$.

\subsection{Pose estimation errors}

We rotate the wooden arm by a continuous rotation of $\theta=120^{\circ}$ with a velocity of $10^{\circ} / \mathrm{s}$. The sample rates of the MPU6050 and the ISNC02 are $200 \mathrm{~Hz}$ and $166 \mathrm{~Hz}$ respectively.

The acquired data are converted into physical units and analyzed using the DR Seq. 2.3.1) and the EKF Seq. (2.3.2) algorithms and then compared to the simulated data. The KFs are tuned empirically. We run 5 similar acquisitions. The absolute maximum mean error (AME) between the simulated data and the estimates and the SDs are presented in Tab. 2 for orientations and in Tab. 3 for positions. For attitude estimates, the different filters perform greatly with an

Table 2 Errors on the attitude estimates for $120^{\circ}$ acquisitions.

\begin{tabular}{lllllll}
\hline Filter & Roll $\phi^{d}\left[{ }^{\circ}\right]$ & Pitch $\theta^{d}\left[{ }^{\circ}\right]$ & \multicolumn{2}{c}{ Heading $\psi^{d}\left[^{\circ}\right]$} \\
\hline & AME & SD & AME & SD & AME & SD \\
ISNC02 with EKF & 0.16 & 0.0018 & 0.12 & 0.0018 & 0.20 & 0.0016 \\
ISNC02 with DR & 0.22 & 0.0020 & 0.07 & 0.0019 & 0.19 & 0.0027 \\
MPU6050 with EKF & 0.27 & 0.0029 & 0.13 & 0.0021 & 0.25 & 0.0032 \\
MPU6050 with DR & 0.29 & 0.0032 & 0.17 & 0.0022 & 0.27 & 0.0035 \\
\hline
\end{tabular}

$\mathrm{AME}<0.3^{\circ}$ for a $120^{\circ}$ gantry angle. However, differences can be seen when we have discontinuous motions. In this case, the most accurate estimates are made by the ISNC02 (AME of $0.12^{\circ}$ ) and the EKF for MPU6050 (AME of $0.16^{\circ}$ ) compared with the DR for MPU6050 (AME of $0.41^{\circ}$ ) for a given acquisition. For position estimates, the MPU6050 (AME of $\approx 1 \mathrm{~cm}$ for the EKF and AME of 
Table 3 Errors on the position estimates for $120^{\circ}$ acquisitions.

\begin{tabular}{lllllll}
\hline Filter & ${ }^{d} p_{x}[\mathrm{~mm}]$ & ${ }^{d} p_{y}[\mathrm{~mm}]$ & \multicolumn{3}{c}{${ }^{d} p_{z}[\mathrm{~mm}]$} \\
\hline & AME & SD & AME & SD & AME & SD \\
ISNC02 with EKF & 2.9 & 0.032 & 1.7 & 0.025 & 2.9 & 0.036 \\
ISNC02 with DR & 3.0 & 0.039 & 1.6 & 0.039 & 3.2 & 0.041 \\
MPU6050 with EKF & 12 & 0.097 & 9.1 & 0.080 & 14 & 0.093 \\
MPU6050 with DR & 96 & 0.093 & 61 & 0.084 & 102 & 0.10 \\
\hline
\end{tabular}

$\approx 1 d m$ for the DR) performs poorly compared to the ISNC02 (AME $\leq 3.2 \mathrm{~mm}$ for both algorithms). However, the EKF appears to reduce the tracking error of the C-arm equipped with the poorly calibrated IMUs by $\approx 80 \%$. Note that the pose estimation errors increases with the acquisition time.

\subsection{D-projections errors}

In order to propagate the attitude estimation errors to the $2 \mathrm{D}$ projections, we simulate the following scenario. We project a square grid of 5 by 5 markers on the detector screen. The source-to-detector distance is $s d d=1.5 \mathrm{~m}$ and the source-to-grid distance is $s w d=0.7 \mathrm{~m}$ as shown in Fig. 7. We empirically choose $|s x-d x|=|s y-d y|=0.1 \mathrm{~m}$. In order to compare the influence of the

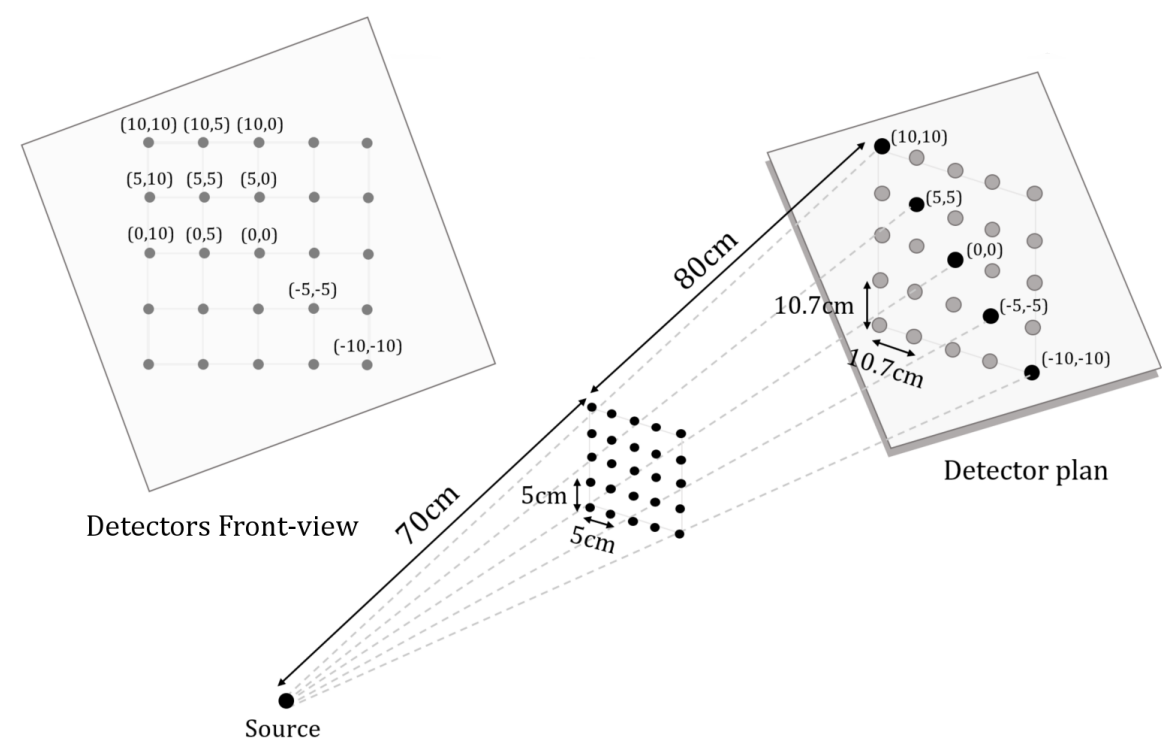

Fig. 7 The simulation scenario for error propagation. $(0,0)$ represents the center point of the grid and $(10,10)$ represents the upper left corner. The projection magnification factor is 2.14 . 
errors on the different projection matrix parameters, we simulate comparable and significant uncertainty values of these parameters: $\delta^{d} \phi=\delta^{d} \theta=\delta^{d} \psi=$ $0.075 \operatorname{rad}\left(\approx 4.3^{\circ}\right)$ and $\delta s x=\delta s y=\delta d x=\delta d y=\delta s d d=\delta s w d=50 \mathrm{~mm}$. The error is propagated for one parameter at a time using the (19). We display the obtained error bars for some geometric parameters in Fig. 8. The errors on $d x$ and $s x$ lead to horizontal error bars on the 2D projections of lengths of $35 \mathrm{~mm}$ and $40 \mathrm{~mm}$ respectively. When the errors on $d y$ and $s y$ lead to vertical error bars of the same lengths (35 $\mathrm{mm}$ and $40 \mathrm{~mm}$ respectively). An error on swd propagate as a uniform error of $(5 \mathrm{~mm}, 5 \mathrm{~mm})$ on the grid. The first term of the parenthesis refers to the length of the error bar according to ${ }^{d} \mathbf{x}$ and the second to the length according to ${ }^{d} \mathbf{y}$. The errors induced by the other parameters as a function of the grid point are indicated in Tab. 4.

The errors on the detector angles and the out-plane translations depend on

Table 4 The errors obtained for different points of the projected grid depending on the error for a given parameter. All the errors are given in $m m$ and in the form $(a, b)$, where $a$ and $b$ are the lengths of the error bars according to ${ }^{d} \mathbf{x}$ and to ${ }^{d} \mathbf{y}$ respectively.

\begin{tabular}{lllll}
\hline Point & $s d d$ & $d_{\phi}$ & $d_{\theta}$ & ${ }^{d} \psi$ \\
\hline$(0,0)$ & $(0,0)$ & $(0,0)$ & $(0,0)$ & $(0,0)$ \\
$(0,5)$ & $(0,2.5)$ & $(0,0)$ & $(0.39,0.39)$ & $(0,0.39)$ \\
$(0,10)$ & $(0,5)$ & $(0,0)$ & $(0.79,0.79)$ & $(0,0.79)$ \\
$(5,0)$ & $(2.5,0)$ & $(0.39,0.39)$ & $(0,0)$ & $(0.39,0)$ \\
$(5,5)$ & $(2.5,2.5)$ & $(0.39,0.39)$ & $(0.39,0.39)$ & $(0.39,0.39)$ \\
$(5,10)$ & $(2.5,5)$ & $(0.39,0.39)$ & $(0.79,0.79)$ & $(0.39,0.79)$ \\
$(10,0)$ & $(5,0)$ & $(0.79,0.79)$ & $(0,0)$ & $(0.79,0)$ \\
$(10,5)$ & $(5,2.5)$ & $(0.79,0.79)$ & $(0.39,0.39)$ & $(0.79,0.39)$ \\
$(10,10)$ & $(5,5)$ & $(0.79,0.79)$ & $(0.79,0.79)$ & $(0.79,0.79)$ \\
\hline
\end{tabular}

the values of the differences $|s x-d x|$ and $|s y-d y|$. Under experimental conditions, the errors obtained for the different detector angles are comparable. The impacts of the out-plane translations sdd and sid are also similar. The errors for the detector angles and $s d d$ increase as we move from the piercing point (here located in $(0.07 \mathrm{~m}, 0.07 \mathrm{~m})$ ) to grid borders. These parameters have also little influence on the 2D projections compared to the in-plane translations $d x$, $d y, s x$ and $s y$. If we propagate the errors obtained using the EKF algorithm in Seq. 3.3, we obtain a total absolute maximum error on the 2D projections of $(7.4 \mathrm{~mm}, 4.7 \mathrm{~mm})$ for the ISNC02 and of $(29 \mathrm{~mm}, 23 \mathrm{~mm})$ for the MPU6050. They are displayed in Fig. 9.

\section{Conclusions and discussion}

During a continuous rotation of the C-arm mock-up, the required angle accuracy by our CBCT algorithms is reached by the three-axis gyroscopes from the two brands (absolute mean error $<0.3^{\circ}$ for all sensors). Taking into account a dynamic model of the $\mathrm{C}$-arm provides slightly better attitude estimates. 

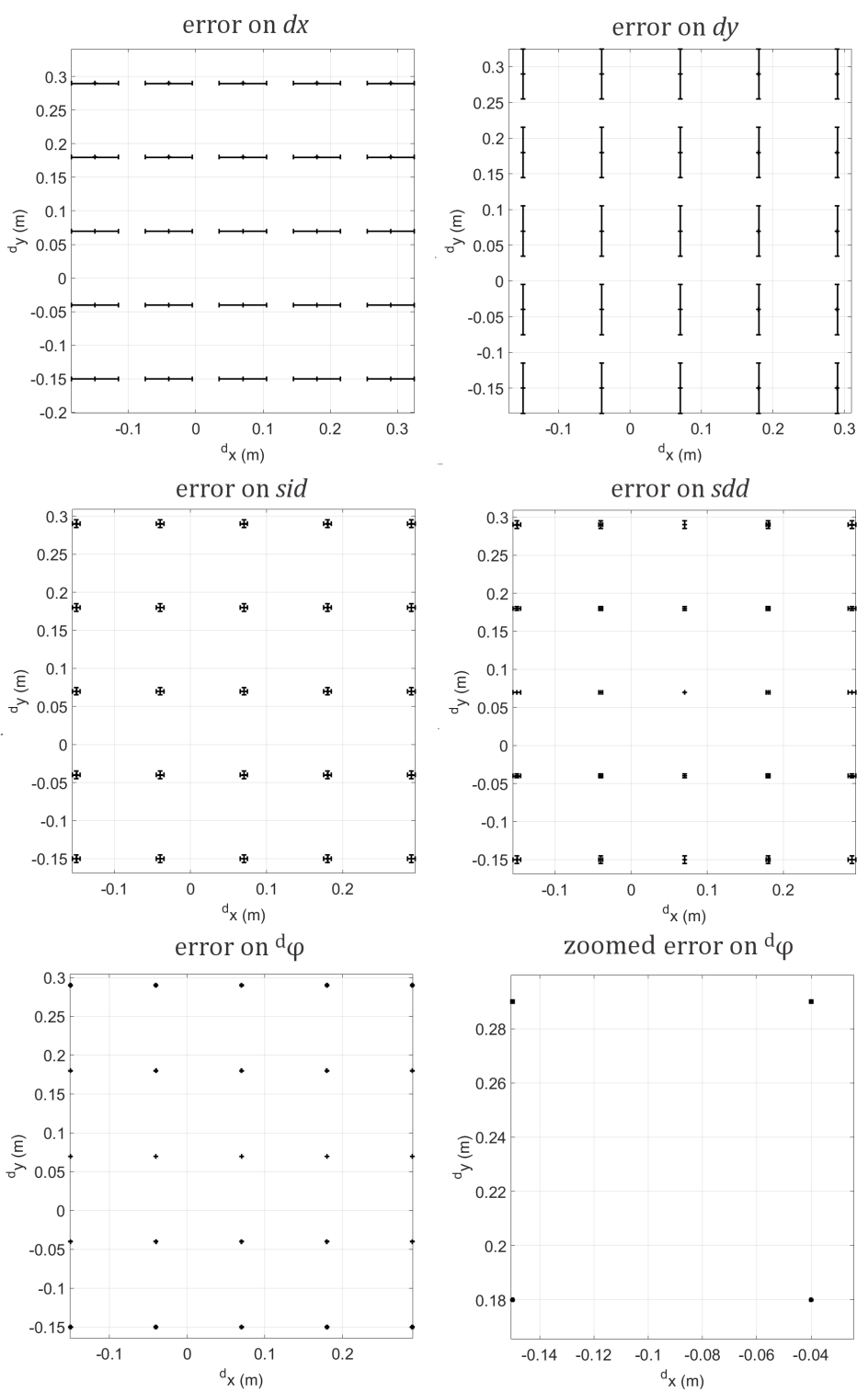

Fig. 8 Impact of the errors on different projection parameters on the $2 \mathrm{D}$ projected grid.

For position estimation, the maximum accuracy is reached for the factory calibrated sensors (position error $\leq 3.2 \mathrm{~mm}$ ). It is not sufficient for CBCT which requires a precision of $0.1 \mathrm{~mm}$ to $0.5 \mathrm{~mm}$. However, it can be suitable for other clinical uses where a high spatial accuracy is needed such as $2 \mathrm{D} / 3 \mathrm{D}$ registration. The inexpensive and manually calibrated accelerometer performs poorly with an error $>1 \mathrm{~cm}$. Therefore, sensors calibration appears to be crit- 

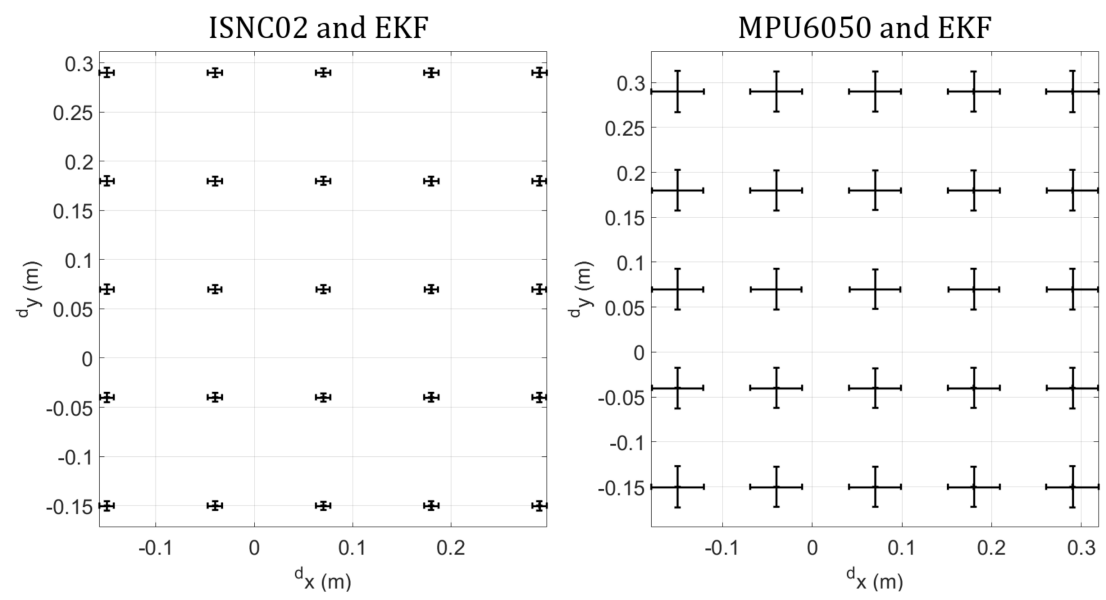

Fig. 9 Impact of the pose estimation errors for a continuous rotation of the rotated-arm by $120^{\circ}$ on the $2 \mathrm{D}$ projections.

ical in order to achieve sufficient accuracy. Taking into account a suitable dynamic model appears to slightly reduce the tracking errors of the C-arm equipped with the accurately calibrated accelerometers. It also reduces by $\approx 80 \%$ the tracking errors of the biased ones. However, in order to achieve a sufficient accuracy for CBCT while using simple pose estimation algorithms the measurement errors must not exceed $1.5 \times 10^{-4} \mathrm{~m} / \mathrm{s}^{2}$ during the scan. The parameters of the C-arm's projective geometry that have the greatest impact on the 2D projections are the in-plane translations of the X-ray source $s x$ and $s y$ and of the detector $d x$ and $d y$. These translations determine the position of the piercing point $[s x-d x \quad s y-d y]$ and uniformly affect the entire projected image. Therefore, the in-plane translations are the most critical parameters to be efficiently tracked in order to avoid artifacts in the $3 \mathrm{D}$ volume reconstruction. Compared to the in-plane translations, the out-plane translations swd and $s d d$ and the orientation of the detector have little influence on the 2D projections. Since CBCT is performed off-line, it can be interesting to implement a smoother such as Rauch-Tung-Striebel [3] rather than a filter in order to achieve a better accuracy. It can also be interesting to improve the dynamic models or to fuse the information from multiple inertial sensors.

\section{Disclosures}

Funding: This study was funded by Thales AVS France and the ANRT (Association Nationale Recherche Technologie).

Conflict of Interest: The authors declare that they have no conflict of interest. This article does not contain any studies with human participants performed by any of the authors. This articles does not contain patient data. 


\section{References}

1. Anderson BD, Moore JB (1979) Optimal filtering. Englewood Cliffs 21:2295

2. Chapra SC, Canale RP (1998) Numerical methods for engineers, vol 2. Mcgraw-hill New York

3. Chiang KW, Duong TT, Liao JK, Lai YC, Chang CC, Cai JM, Huang SC (2012) On-line smoothing for an integrated navigation system with low-cost MEMS inertial sensors. Sensors 12(12):17372-17389

4. Cho Y, Moseley DJ, Siewerdsen JH, Jaffray DA (2005) Accurate technique for complete geometric calibration of cone-beam computed tomography systems. Medical physics 32(4):968-983

5. Daly MJ, Siewerdsen JH, Cho YB, Jaffray DA, Irish JC (2008) Geometric calibration of a mobile C-arm for intraoperative cone-beam CT. Medical physics 35(5):2124-2136

6. Forsyth DA, Ponce J (2003) A modern approach. Computer vision: a modern approach pp 88-101

7. Grewal M, Andrews A (2010) How good is your gyro [ask the experts]. IEEE Control Systems 30(1):12-86

8. Grzeda V, Fichtinger G (2010) C-arm rotation encoding with accelerometers. International journal of computer assisted radiology and surgery $5(4): 385-391$

9. Inven Sense Inc (2013) MPU-6000 and MPU-6050 product specitication revision 3.4. Document Number: PS-MPU-6000A-00

10. Jain AK, Mustafa T, Zhou Y, Burdette C, Chirikjian GS, Fichtinger G (2005) FTRAC - a robust fluoroscope tracking fiducial. Medical Physics 32:3185-3198, URL https://doi.org/10.1118/1.2047782

11. Jia YB (2008) Quaternions and rotations. Com S 477(577):15

12. Khoury A, Whyne CM, Daly M, Moseley D, Bootsma G, Skrinskas T, Siewerdsen J, Jaffray D (2007) Intraoperative cone-beam CT for correction of periaxial malrotation of the femoral shaft: A surface-matching approach. Medical physics 34(4):1380-1387

13. Mitschke M, Navab N, Schütz O (2000) Online geometrical calibration of a mobile C-arm using external sensors. In: SPIE Medical Imaging, vol 3976, pp 580-587, URL https://www.researchgate.net/publication/252962816

14. Navab N, Bani-Hashemi A, Nadar MS, Wiesent K, Durlak P, Brunner T, Barth K, Graumann R (1998) 3D reconstruction from projection matrices in a c-arm based 3D-angiography system. In: International Conference on Medical Image Computing and Computer-Assisted Intervention, Springer, pp 119-129

15. Orth RC, Wallace MJ, Kuo MD, Technology Assessment Committee of the Society of Interventional Radiology (2008) C-arm cone-beam CT: general principles and technical considerations for use in interventional radiology. Journal of Vascular and Interventional Radiology 19(6):814-820

16. Siewerdsen J, Moseley D, Burch S, Bisland S, Bogaards A, Wilson B, Jaffray D (2005) Volume CT with a flat-panel detector on a mobile, isocentric 
c-arm: Pre-clinical investigation in guidance of minimally invasive surgery. Medical physics 32(1):241-254

17. Smith L, Pleasance M, Seeton R, Archip N, Rohling R (2005) Automatic detection of fiducial markers in fluoroscopy images for on-line calibration. Medical physics 32(6Part1):1521-1523

18. Strobel NK, Heigl B, Brunner TM, Schuetz O, Mitschke MM, Wiesent $\mathrm{K}$, Mertelmeier T (2003) Improving 3D image quality of X-ray C-arm imaging systems by using properly designed pose determination systems for calibrating the projection geometry. In: Medical Imaging 2003: Physics of Medical Imaging, International Society for Optics and Photonics, vol 5030, pp 943-955

19. Tang TSY, MacIntyre NJ, Gill HS, Fellows RA, Hill N, Wilson DR, Ellis RE (2004) Accurate assessment of patellar tracking using fiducial and intensity-based fluoroscopic techniques. Medical image analysis 8(3):343351

20. Thales Visionix Inc (2015) Navchip precision 6-axis MEMS inertial measurement unit

21. Thürauf S, Körner M, Vogt F, Hornung O, Nasseri MA, Knoll A (2017) Environment effects at phantom-based X-ray pose measurements. In: Engineering in Medicine and Biology Society (EMBC), 2017 39th Annual International Conference of the IEEE, IEEE, pp 1836-1839

22. Wolff T, Lasso A, Eblenkamp M, Wintermantel E, Fichtinger G (2014) C-arm angle measurement with accelerometer for brachytherapy: an accuracy study. International journal of computer assisted radiology and surgery $9(1): 137-144$

23. Zhang M, Zaider M, Worman M, Cohen G (2004) On the question of 3D seed reconstruction in prostate brachytherapy: the determination of x-ray source and film locations. Physics in Medicine \& Biology 49(19):N335 